\title{
Analgesia preventiva en pacientes sometidos a cirugía abierta por reparación del manguito rotador
}

\author{
Preventive analgesia in patients undergoing \\ open surgery for rotator cuff repair
}

\author{
Martínez-Domínguez JM, ${ }^{*}$ Quiroz-Williams J, ${ }^{\star}$ Quíntela-Núñez del Prado $\mathrm{H}^{\S}$ \\ Hospital de Traumatología de la Unidad Médica de Alta Especialidad \\ «Dr. Victorio de la Fuente Narváez», Instituto Mexicano del Seguro Social.
}

RESUMEN. Introducción: La analgesia preventiva en cirugía ortopédica cada vez toma mayor importancia debido a que el control del dolor postquirúrgico es más grato para el paciente y le permite iniciar rehabilitación de forma temprana. Con esta premisa nos planteamos la siguiente hipótesis. La percepción del dolor postquirúrgico a las dos, 12 y 24 horas con infiltración local en la herida quirúrgica con ropivacaína más analgesia sistémica será menor en comparación con los pacientes que se infiltren con solución salina en la herida quirúrgica más analgesia sistémica. Material y métodos: Se realizó un ensayo clínico controlado aleatorizado doble ciego, prospectivo en el período comprendido de Enero de 2018 a Agosto de 2018. Resultados: Se rechazó la hipótesis nula en el grupo valorado a las dos horas y se aceptó la hipótesis nula en los grupos valorados a las 12 y 24 horas. Conclusión: La infiltración con ropivacaína es un método efectivo para el manejo del dolor postoperatorio durante las primeras dos horas en procedimientos de cirugía abierta de hombro.

Palabras clave: Analgesia, cirugía, ropivacaína, infiltración, dolor.
ABSTRACT. Introduction: Preventive analgesia in orthopedic surgery is becoming increasingly important because post-surgical pain management is more pleasant for the patient and allows them to initiate rehabilitation early. With this premise we consider the following hypothesis. The perception of post-surgical pain at two, 12 and 24 hours with local infiltration into the surgical wound with ropivacaine plus systemic analgesia will be less compared to patients who infiltrate with saline into the surgical wound plus systemic analgesia. Material and methods: A randomized double-blind, prospective randomized controlled clinical trial was conducted in the period January 2018 to August 2018. Results: The null hypothesis is rejected in the group valued at two hours and the null hypothesis is accepted in the groups valued at 12 and 24 hours. Conclusion: Ropivacaine infiltration is an effective method for managing postoperative pain for the first 2 hours in open shoulder surgery procedures.

Keywords: Analgesia, surgery, ropivacaine, infiltration, pain.

\section{Nivel de evidencia: I}

* Jefe de residente de la Especialidad de Traumatología y Ortopedia, Hospital de Traumatología.

* Jefe de la División de Investigación en Salud, Instituto Mexicano del Seguro Social.

$\S$ Jefe de Servicio de Cirugía Articular Reconstructiva, Instituto Mexicano del Seguro Social.

Unidad Médica de Alta Especialidad (UMAE) «Dr. Victorio de la Fuente Narváez».

Correspondencia:

José Manuel Martínez Domínguez

Hospital de Traumatología de la UMAE «Dr. Victorio de la Fuente Narváez», IMSS.

Av. Colector $15 \mathrm{~s} / \mathrm{n}$ Esq. Av. Instituto Politécnico Nacional, Col. Magdalena de las Salinas, C.P. 07760. Alcaldía Gustavo A. Madero. CDMX.

E-mail: manuel.ortopedia@outlook.com 


\section{Introducción}

El dolor es una respuesta adaptativa que alerta al organismo. Puede proporcionar información de situaciones que dañan la integridad de la homeostasia de los sistemas del cuerpo humano o situaciones que atentan contra la vida. Sin embargo, el dolor postquirúrgico se considera una respuesta maladaptativa. ${ }^{1}$ Tal respuesta no aporta al paciente ningún beneficio y puede traer consecuencias negativas, tales como prolongar la estancia intrahospitalaria, prolongar el inicio de la rehabilitación y aumentar el riesgo de infección. ${ }^{2}$ El dolor agudo después de cirugía es un importante factor predictivo del dolor crónico postquirúrgico, el cual se ha detectado en 10-65\% de los pacientes después de cirugía., Además, hemos observado mala evolución postquirúrgica en los pacientes que han mostrado limitación de rangos de movimiento e incluso capsulitis adhesiva. Al reducir el dolor postquirúrgico el paciente tendrá mayor satisfacción de su procedimiento, facilidad para iniciar ejercicios de rehabilitación, estancia intrahospitalaria más corta y menos complicaciones. ${ }^{5}$ En la literatura actual los medicamentos para control del dolor postquirúrgico son los opiáceos. Estos analgésicos pueden traer al paciente distintas complicaciones, las cuales son náusea, vómito, prurito, entre otras. Este grupo de medicamentos ha resultado deficiente en el control del dolor postquirúrgico, ${ }^{6,7}$ por lo que la analgesia preventiva pretende lograr un mejor control del dolor. Dicha analgesia es el uso de medicamentos previos al estímulo doloroso o en este caso previo al despertar de la cirugía. El concepto fue propuesto en 1980 en estudios experimentales que mostraron que las medidas para antagonizar los receptores de nocicepción antes de que éstos recibieran un estímulo, prevenían la hipersensibilización reduciendo la intensidad del dolor después de la lesión o cirugía. ${ }^{8}$ Esta medida anticipatoria disminuye los cambios de los procesos sensitivos a nivel central, los cuales son responsables de hiperalgesia y alodinia. ${ }^{9}$

Las intervenciones invasivas provocan la liberación de histamina y mediadores inflamatorios como prostaglandinas y bradiquininas en el sitio de incisión que estimulan los receptores neurales. La señal de nocicepción se transmite a través de la médula espinal por el cuerno posterior. El receptor de n-metil-d-aspartate (NMDA) en la médula espinal es exclusivamente para el control de los estímulos dolorosos. La persistencia de la liberación de mediadores inflamatorios sensibiliza la función de los receptores nociceptivos. Dicha sensibilización disminuye el umbral de disparo y aumenta el rango de respuesta basal de descarga. ${ }^{10,11}$ La analgesia multimodal preventiva influye en todos los componentes en la vía mencionada. ${ }^{12}$

La ropivacaína es un enatiómero puro, su estructura química proviene de la bupivacaína y la mepivacaína. Sin embargo, este analgésico tiene un rango de seguridad más amplio en el sistema nervioso y cardiovascular, por lo que resulta más favorable debido a una menor lopisolubilidad y efecto vasoconstrictor. ${ }^{13}$ Es metabolizado principalmente por el CYP1A2. ${ }^{14}$ Se ha estudiado el medicamento en infil- tración de heridas quirúrgicas en una concentración de 300 y $375 \mathrm{mg}$ presentando una eficacia similar. Se observó que la concentración máxima plasmática fue de $30 \mu \mathrm{g} / \mathrm{ml}$ en un paciente a quien se le administró la dosis de $375 \mathrm{mg}$, por lo que se concluyó que el uso de dicho medicamento es seguro cuando se infiltra en heridas. ${ }^{15}$

La incidencia de dolor de hombro en la población general es de alrededor de 11.2 casos por 1,000 pacientes por año y el manguito rotador es la causa principal de dolor del hombro. ${ }^{16}$ Es conocido que las afecciones del mango rotador aumentan con el paso del tiempo, ya que tienen una relación directa con un proceso de deterioro progresivo más que con un evento traumático único y específico. Las lesiones del manguito rotador tienen un impacto en la calidad de vida y en la discapacidad de los pacientes que es equiparable a aquéllos que tienen diabetes, infarto al miocardio, insuficiencia cardíaca congestiva o depresión. ${ }^{17}$ Esta enfermedad, tal y como fue interpretada por Neer, es probablemente la causa más habitual de dolor localizado en la región anteroexterna del hombro. El manguito de los rotadores tiene la función de estabilizar la cabeza del húmero mientras el músculo deltoides desarrolla la abducción del hombro. Por este motivo, si se produce una lesión del mismo la consecuencia puede ser un grave deterioro funcional. En la literatura médica se han apuntado dos tipos de factores distintos posiblemente implicados en su producción: intrínsecos y extrínsecos. Entre los intrínsecos hay que mencionar el deterioro degenerativo del tendón. Se sugiere que la degeneración tendinosa es la causa primaria del desgarro parcial del manguito que permite la migración proximal de la cabeza humeral, lo que a su vez da como resultado el conflicto subacromial y el aumento de fricción en la zona que conduce a la ruptura completa del tendón. En lo que respecta a los factores extrínsecos, la forma, el tamaño y sobre todo la prominencia anterior excesiva del acromion son los principales implicados en el origen de esta lesión. $\mathrm{Su}$ tratamiento quirúrgico debe ir dirigido a descomprimir y reconstruir el manguito rotador desgarrado de la manera más completa posible para recuperar el equilibrio de fuerzas y la función del hombro. ${ }^{18}$

\section{Material y métodos}

Se realizó un ensayo clínico controlado aleatorizado doble ciego en el período comprendido de Enero de 2018 a Agosto de 2018 con un tamaño de muestra de 114 pacientes con edades comprendidas entre 41 y 70 años con diagnóstico clínico radiológico de síndrome de pinzamiento subacromial y ruptura del manguito de los rotadores del hombro tratados con cirugía abierta de reparación del manguito rotador, bursectomía y descompresión subacromial. Se excluyó a los pacientes a quienes se les realizó un bloqueo interescalénico y lesiones masivas del manguito rotador a los cuales no fue posible realizar reparación de los mismos.

Los pacientes se clasificaron en dos grupos de forma aleatoria, cada uno compuesto por 57 pacientes. En todos los ca- 
sos se practicó el mismo manejo anestésico que consistió en anestesia general con intubación orotraqueal, en los cuales se utilizaron los siguientes medicamentos: propofol, lidocaína, atracurio, fentanil, midazolam y sevofluorano. A los pacientes del primer grupo se les administraron $10 \mathrm{~cm}^{3}$ de solución fisiológica al finalizar el procedimiento quirúrgico y cierre de herida. A unos cuantos segundos se les administró ropivacaína $7.5 \mathrm{mg} / \mathrm{ml} 10 \mathrm{~cm}^{3}$, el equivalente a $75 \mathrm{mg}$ de ropivacaína. Todas las soluciones fueron preparadas por el departamento de anestesiología bajo instrucción de la aleatorización.

En el control del dolor postquirúrgico se indicó en la nota postquirúrgica un gramo de paracetamol vía oral y rescates con ketorolaco $30 \mathrm{mg}$ intravenosos en caso de reportar dolor mayor de seis acorde a la escala verbal análoga del dolor. Se midió la intensidad del dolor en todos los pacientes a las dos, 12 y 24 horas después de la cirugía con la aplicación de pain scale your digital log for chronic pain instalada en una tableta electrónica, con ausencia de dolor y con 10 dolor severo. Se recopiló la información en la base de datos y se analizaron los resultados.

\section{Resultados}

La muestra del grupo 1 concluyó con un total de 57 pacientes, conformada por 34 mujeres y 23 hombres, de los cuales en porcentaje corresponde a 59 y $41 \%$ respectivamente. La edad máxima fue de 70 años y la mínima de 49 años con una mediana de 60 . El lado derecho e izquierdo fueron afectados en 59\% (33 pacientes) y $41 \%$ ( 24 pacientes) respectivamente. Se observó que la lesión del manguito rotador fue pequeña en 12 pacientes $(22 \%)$ y masiva en 14 pacientes $(25 \%)$.

La muestra del grupo 2 concluyó con un total de 57 pacientes, conformada por 36 mujeres y 21 hombres, de los cuales en porcentaje corresponde a 63 y $37 \%$ respectivamente. La edad máxima fue de 70 años y la mínima de 41 años con una mediana de 60 . El lado derecho e izquierdo fueron afectados en 64\% (36 pacientes) y $36 \%$ (21 pacientes) respectivamente. Se observó que la lesión del manguito fue pequeña en 19 pacientes (33\%), mediana en $14(25 \%)$, grande en nueve ( $9 \%$ ) y masiva en 15 pacientes (26\%).

Se analizaron los datos con chi chuadrada y se obtuvo el valor de $\mathrm{p}$. La medición del dolor a las dos horas fue menor comparada con el grupo control, mostrando diferencia estadísticamente significativa $(\mathrm{p}<0.01)$, por lo que se rechazó la hipótesis nula en el grupo valorado a las dos horas y se aceptó la hipótesis nula en los grupos valorados a las 12 y 24 horas.

\section{Discusión}

La cirugía abierta de hombro es un procedimiento común en nuestro hospital. La reparación de manguito rotador es un procedimiento que cursa con considerable dolor postquirúrgico, poniendo en riesgo al paciente a una mala evolución y a una rehabilitación fallida. En un esfuerzo por disminuir el dolor postquirúrgico, se decidió investigar en la analgesia preventiva con ropivacaína aplicada al final de la cirugía en el sitio de abordaje.

La duración de la analgesia preventiva es probablemente multifactorial dependiendo del cuidado de tejidos blandos en el transquirúrgico, duración de la cirugía y umbral del dolor. Nuestro resultado mostró la eficacia de la analgesia preventiva en la cirugía abierta de hombro para reparación de manguito rotador durante las primeras dos horas en el postquirúrgico. Sin embargo, no hay todavía información en la literatura respecto a la analgesia preventiva aplicada directamente en el sitio quirúrgico que valide el uso de la misma ni estudios de costo beneficio donde se obtenga una ventaja económica.

\section{Conclusión}

La analgesia preventiva es un método efectivo para el manejo del dolor postoperatorio durante las primeras dos horas en procedimientos de cirugía abierta de hombro donde se realice reparación del manguito rotador. Desde el punto de vista estadístico, el dolor es significativamente menor a las dos horas en comparación con el grupo control. A las 12 y 24 horas postquirúrgicas no hay diferencia en la percepción del dolor.

\section{Referencias}

1. Amiri HR, Mirzaei M, Taghi M, Mohammadi B, Tavakoli F. Multimodal preemptive analgesia with pregabalin, acetaminophen, naproxen, and dextromethorphan in radical neck dissection surgery: a randomized clinical trial. Anesth Pain Med. 2016; 6 (4): e33526. Available from: https://www.ncbi.nlm.nih.gov/pmc/articles/ PMC5099949/pdf/aapm-06-04-33526.pdf.

2. Brunicardi FC, Schwartz SI. Schwartz's principles of surgery. $8^{\text {th }}$ ed. New York; London: McGraw-Hill; 2005.

3. Kehlet H, Jensen TS, Woolf CJ. Persistent postsurgical pain: risk factors and prevention. Lancet. 2006; 367(9522): 1618-25. Available from: http://www.ncbi.nlm.nih.gov/pubmed/16698416.

4. Macrae WA. Chronic pain after surgery. Br J Anaesth. 2001; 87(1): 8898. Available from: http://www.ncbi.nlm.nih.gov/pubmed/11460816.

5. Amruta VD, Rupesh T. Utilization of analgesics in perioperative cases of teaching hospital. Int J Med Pharm Sci. 2013; 3(6): 14-9. Available from: http://www.scopemed.org/?mno=34155.

6. Apfelbaum JL, Chen C, Mehta SS, Gan TJ. Postoperative pain experience: results from a national survey suggest postoperative pain continues to be undermanaged. Anesth Analg. 2003; 97(2): 534 40, table of contents. Available from: http://www.ncbi.nlm.nih.gov/ pubmed/12873949.

7. Gan TJ, Habib AS, Miller TE, White W, Apfelbaum JL. Incidence, patient satisfaction, and perceptions of post-surgical pain: results from a US national survey. Curr Med Res Opin. 2014; 30(1): 149-60. Available from: http://www.tandfonline.com/doi/full/10.1185/030079 95.2013.860019

8. Woolf CJ. Evidence for a central component of post-injury pain hypersensitivity. Nature. 1983; 306(5944): 686-8. Available from: http://www.ncbi.nlm.nih.gov/pubmed/6656869.

9. Hariharan S, Moseley H, Kumar A, Raju S. The effect of preemptive analgesia in postoperative pain relief. A prospective double-blind randomized study. Pain Med. 2009; 10(1): 49-53. Available from: https://watermark.silverchair.com/10-1-49.pdf?token=AQECA Hi208BE49Ooan9kkhW_Ercy7Dm3ZL_9Cf3 qfKAc485 ysgAAAdwwggHYBgkqhkiG9w0BBwagggHJMIIBxQIBADCCA b4GCSqGSIb3DQEHATAeBglghkgBZQMEAS4wEQQMnpcje 
APyqIIOmH6_AgEQgIIBj3WyYmv3URV38EOHhXguD4oPubguRu 7blqefluPzBEeeBki

10. Carr DB, Goudas LC. Acute pain. Lancet. 1999; 353(9169): 2051-8. Available from: http://www.ncbi.nlm.nih.gov/pubmed/10376632.

11. Julius D, Basbaum AI. Molecular mechanisms of nociception. Nature. 2001; 413(6852): 203-10. Available from: http://www.nature.com/ doifinder/10.1038/35093019.

12. Gandhi K, Viscusi E. Multimodal pain management techniques in hip and knee arthroplasty. J New York Sch Reg Anesth. 2009; Available from: https://www.nysora.com/files/2013/pdf/(v13p1-10) MultimodalPainManagement.pdf.

13. Eidy M, Fazel MR, Janzamini M, Haji RM, Moravveji AR. Preemptive analgesic effects of transcutaneous electrical nerve stimulation (TENS) on postoperative pain: a randomized, double-blind, placebo-controlled trial. Iran Red Crescent Med J. 2016; 18(4): e35050. Available from: http://www.ncbi.nlm.nih.gov/pubmed/27275401.

14. Markham A, Faulds D. Ropivacaine. A review of its pharmacology and therapeutic use in regional anaesthesia. Drugs. 1996; 52(3): 429-49. Available from: http://link.springer.com/10.2165/00003495199652030-00012.
15. Pettersson N, Emanuelsson BM, Reventlid H, Hahn RG. High-dose ropivacaine wound infiltration for pain relief after inguinal hernia repair: a clinical and pharmacokinetic evaluation. Reg Anesth Pain Med. 1998; 23(2): 189-96. Available from: http://journals.lww.com/ $\mathrm{rapm} /$ pages/articleviewer.asp $\mathrm{P}$ year $=1998 \&$ issue $=23020 \&$ article $=00$ 013\&type $=$ abstract

16. Clayton RAE, Court-Brown CM. The epidemiology of musculoskeletal tendinous and ligamentous injuries. Injury. 2008; 39(12): 1338-44. Available from: http://www.ncbi.nlm.nih.gov/ pubmed/19036362.

17. Piitulainen K, Ylinen J, Kautiainen H, Häkkinen A. The relationship between functional disability and health-related quality of life in patients with a rotator cuff tear. Disabil Rehabil. 2012; 34(24): 2071-5. Available from: http://www.ncbi.nlm.nih. gov/pubmed/22494417.

18. Arenas PA, Ortega AJ, Arenas MA, Ayala PH, Garbayo MA. Impingement syndrome with rotator-cuff tears. Treatment and prognosis. 2005; 40(224): 159-69. Available from: http://www. cirugia-osteoarticular.org/adaptingsystem/intercambio/revistas/ articulos/171_159.pdf. 